\title{
Protection from high-velocity projectiles
}

\author{
A. Gerasimov and S. Pashkov \\ Tomsk State University, Research Institute of Applied Mathematics and Mechanics, 634050 Tomsk, Russia
}

\begin{abstract}
Creation of reliable system of target protection demands research of various ways of counteraction high-speed elongated projectiles. This paper considers the interaction of projectiles with plates and rods thrown towards by explosion. At contact projectiles and rods form a crosswise configuration. Deformation and destruction of projectiles reduce their penetrability and capacity to strike armor-target.
\end{abstract}

\section{The equations describing movement of compressed elastic-plastic body taking into account probabilistic fracture behavior}

The problem is solved in 3-D statement in view of natural heterogeneity of real materials structure affecting distribution of physical-mechanical characteristics along the volume of construction elements and being one of the factors, defining destruction character of the latter.

The necessity to account the given factor for equations of deformable solid mechanics dictates the application of probabilistic laws of physical-mechanical characteristics distribution along the volume of the construction under consideration.

The equations describing spatial adiabatic movement of the strong compressed solid are differential result of fundamental laws of conservation of mass, pulse and energy. In general they have the following form [1-3].

The equation of continuity

$$
\frac{1}{\rho} \frac{d \rho}{d t}+\frac{\partial v_{i}}{\partial x_{i}}=0
$$

The equations of movement

$$
\rho \frac{d v_{i}}{d t}=\rho F_{i}-\frac{\partial P}{\partial x_{i}}+\frac{\partial S_{i j}}{\partial x_{j}}
$$

The equation of energy

$$
\rho \frac{d E}{d t}=S_{i j} \varepsilon_{i j}+\frac{P}{\rho} \frac{d \rho}{d t},
$$

where $x_{i}$ - coordinates; $t$ - time; $\rho_{0}$ - initial density; $\rho$ - current density; $v_{i}$ - velocity vector components; $F_{i}-$ components of the vector of mass forces; $S_{i j}-$ stress deviator components; $E$ - specific internal energy; $\varepsilon_{i j}-$ components of the strain rate tensor; $P$ - pressure.

Equations (1)-(3) must be added by equations that take into account the relevant thermodynamic effects related to the adiabatic compression of the medium and the strength of the medium. In general at action of forces on a solid deformable body both the volume (density) and shape of the body change, and at different dependences. Therefore the tensor of stress is represented as the sum of a pressure and the stress deviator components.

$$
\begin{aligned}
& \sigma_{i j}=S_{i j}-P \delta_{i j}, \quad i, j=1,2,3, \\
& \delta_{i j}=1, \quad i=j, \\
& \delta_{i j}=0, \quad i \neq j,
\end{aligned}
$$

where $\delta_{i j}$ - Kronecker symbol.

To describe resistance of a solid to shear, we use the following ratios:

$$
\begin{gathered}
2 \mu\left(e_{i j}-\frac{1}{3} e_{k k} \delta_{i j}\right)=\frac{D S_{i j}}{D t}+\lambda S_{i j} ; \\
\frac{D S_{i j}}{D t}=\frac{d S_{i j}}{d t}-S_{i k} \omega_{j k}-S_{j k} \omega_{i k} ; \\
2 \omega_{i j}=\frac{\partial v_{i}}{\partial x_{j}}-\frac{\partial v_{j}}{\partial x_{i}} \\
2 e_{i j}=\frac{\partial v_{i}}{\partial x_{j}}+\frac{\partial v_{j}}{\partial x_{i}},
\end{gathered}
$$

and also plasticity condition

$$
J_{2}=\frac{1}{2} S_{i j} S_{i j}=\frac{1}{3} \sigma^{2}
$$

where $e_{i j}$ - strain rate tensor components; $\mu$ - shear modulus; $\sigma$ - dynamic yield stress; $D / D t$ - Jaumann derivative.

The equation of state was chosen in the form of MiGruneisen

$$
P \tilde{A}=E \frac{K \tilde{A}\left(1-{ }_{0} \xi / 2\right)}{(1-c \xi)^{2}} \xi+\rho_{0} \quad{ }_{0} E
$$

where $\tilde{A}_{0}-$ Gruneisen coefficient; $c, K$ - material constants; $\rho_{0}-$ initial density; $\xi=1-\rho_{0} / \rho$.

The limiting equivalent of plastic deformation was used as a criterion of shear fracture [4] $\varepsilon^{p}=\varepsilon_{*}^{p}$. The system of equations (1) - (9) is written in general form for the spatial motion of a deformable body.

The process of destruction of real materials is largely determined by internal structure of the solid, presence of heterogeneity, usually caused by different orientation of the grains in a polycrystalline material or irregularities in 


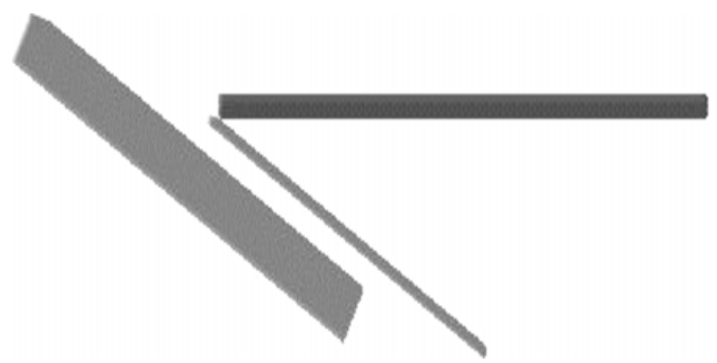

Fig. 1. The initial configuration of the system "target - a throwing plate - projectile".

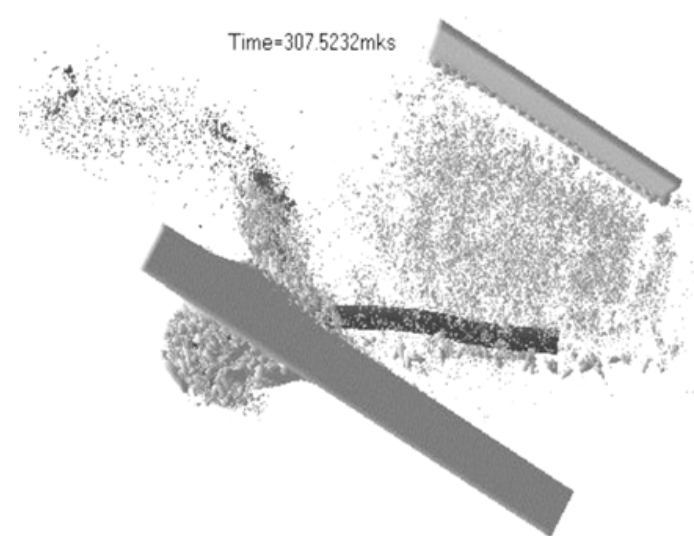

Fig. 2. The three-dimensional picture of projectile interaction with a throwing plate and target.

the composition of composite materials, the difference in micro-strength inside the grain and on inter-grain or interphase boundary. Therefore to the adequacy of the process numerically modeled with the experimental data it is necessary to bring in perturbations to physical-mechanical characteristics of the destroyed environment, i.e. to set casual distribution of the factors determining the strength properties of the material. The natural fragmentation of projectiles and target is calculated by introducing probability distribution mechanism of initial defects in the material structure to describe separated and shear cracks. The reaching its limiting value by equivalent plastic deformation is used as a criterion of fracture under intensive shear deformations.

The initial heterogeneities were modeled by that limiting equivalent plastic deformation was distributed across cells using a modified random number generator issuing a random variable obeying the chosen law of distribution.

The system of the basic equations is supplemented with appropriate initial and boundary conditions. At the initial moment all projectile points have an axial velocity $V_{0}$ with regard to its sign, the plate or rods had normal to the surface of the target velocity $\mathrm{V}_{1}$, the target state is assumed unperturbed. Boundary conditions are as follows: on borders, free from pressure, conditions are satisfied $\sigma_{n}=\tau_{n}=0$. On the contact site between bodies we condition ideal sliding of one material relative to another along the tangent: $\sigma_{n 1}=\sigma_{n 2}, v_{n 1}=v_{n 2}, \tau_{n 1}=\tau_{n 2}=0$, where $\sigma_{n}, \tau_{n}$ - normal and tangent components of stress vector; $v_{n}-$ normal component of velocity vector in a point of contact; indexes 1 and 2 refer to contacting bodies.

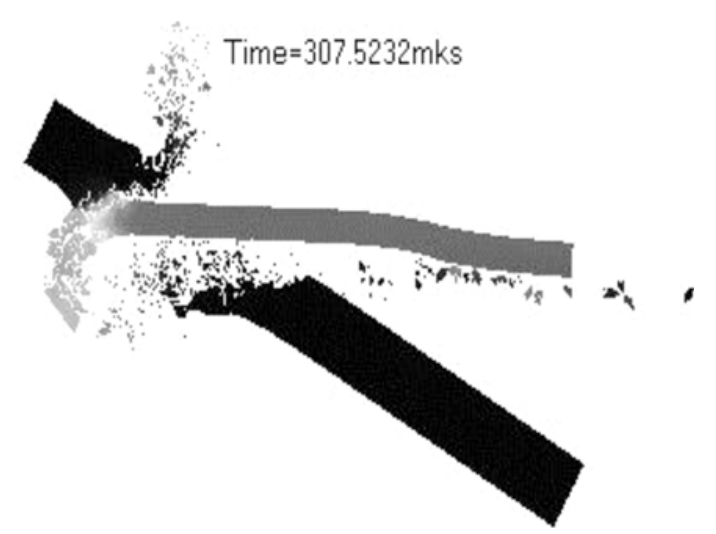

Fig. 3. 2D cross-section of three-dimensional calculated area of projectile interaction with a throwing plate and target.

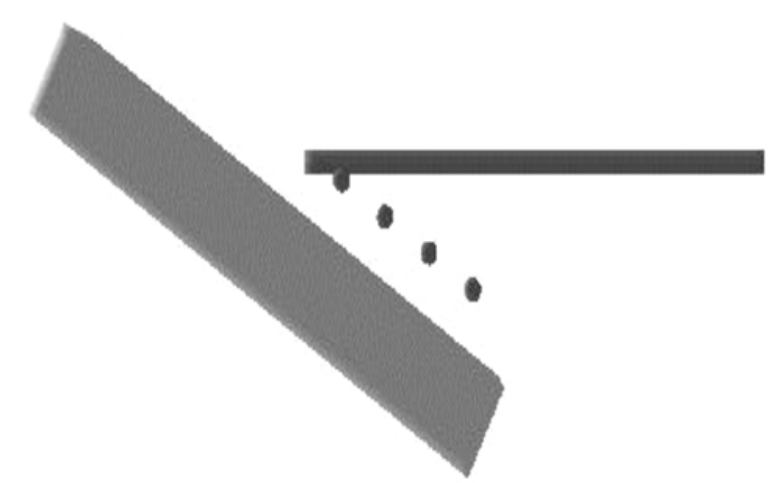

Fig. 4. The initial configuration of the system "target -4 rods projectile.

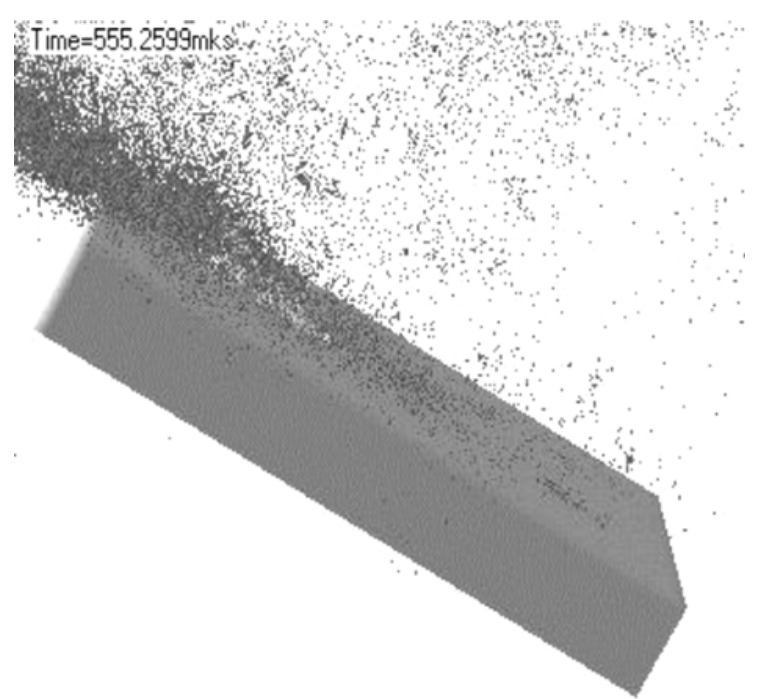

Fig. 5. The three-dimensional picture of projectile interactions with rods and the target.

To calculate elastic-plastic flows one used the technique implemented on tetrahedral cells and based on joint use of Wilkins method for calculating interior points of the body and Johnson method for calculating contact interactions $[2,5,6]$. Three-dimensional area was sequentially split into tetrahedrons by means of automatic grid construction subroutines. 


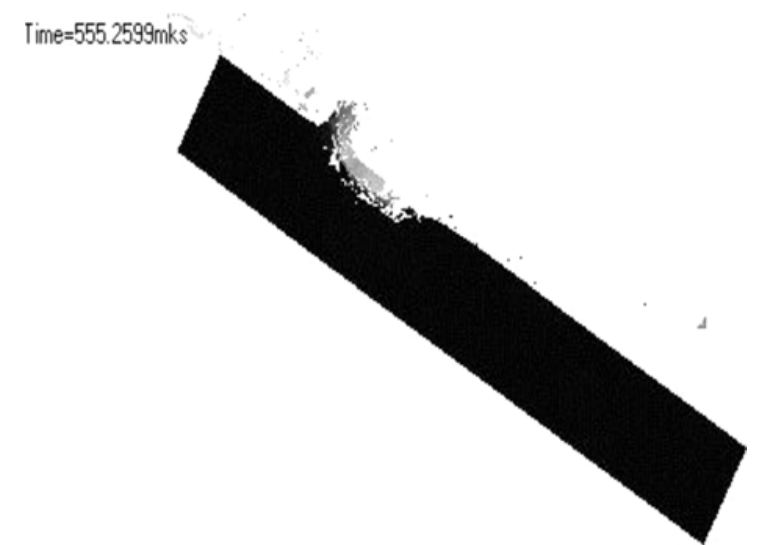

Fig. 6. 2-D cross-section of three-dimensional calculated area of projectile interactions with the rods and the target.

The most complete ideology and methodology of the probabilistic approach to the problem of solids fracture is given in the monograph [7].

\section{The results of calculations}

Two possible impacts on high-speed elongated projectiles of tungsten alloy, approaching the target at a velocity $1500 \mathrm{~km} / \mathrm{sec}$, have been calculated. In the first case steel plate and in the second case four rods of tungsten allow were thrown towards the projectile.

In both cases velocity of the throwing elements was equal to $1000 \mathrm{~m} / \mathrm{sec}$ and was directed along the normal to the target. The thickness of the barrier was $10 \mathrm{~cm}$ and the angel of target deviation from a vertical was $60^{\circ}$ (figure 1).

As is seen from figures 1-3 the plate caused insignificant curvature of the projectile and had no effect on target perforation. Figure 3 demonstrates that by the time equal to 307 microseconds the projectile breaks the target and behind its back surface a significant fragmentation stream is formed. The results of projectile interactions with four tungsten rods are presented in figures 4-6.

A completely different picture is observed when throwing rods of tungsten alloy in projectile. In this case, there is an intense fragmentation of both rods and projectile (figure 5). The remaining projectile tail ricochets from the surface of the target, which has received relatively little damage of the front surface.

\section{Conclusions}

The calculation results presented in figures 1-6 show the undoubted advantage of the second approach to reduce the penetrating power of the projectile. The obtained results confirmed that the proposed approach and the numerical technique developed on its basis enable to simulate processes of targets protection from perforation by highspeed long projectiles in a wide range of speeds and angles of impact, to investigate targets and projectiles fragmentations, and the nature of the forming fragmentation fields.

\section{References}

1. K.P. Stanjukovich (Ed), Physics of explosion (Nauka, Moscow, 1975) [in Russian]

2. M.L. Wilkins, Fundamental Method in Hydrodynamics (Mir, Moscow, 1967) [Russian translation].

3. M.L. Wilkins, Structural impact and crashworthiness (New York, 1984)

4. K.N. Kreyenhagen, M.H. Wagner, J. J. Piechocki, R. L. Bjork, AIAA J. 8, 2147 (1970)

5. G.R. Johnson, D.D. Colby, D.J. Vavrick, Int. J. Numer. Methods Engng., 14, 1865 (1979)

6. G.R. Johnson, Trans. ASME. J. of Appl. Mech., 48, 30 (1981)

7. A.V. Gerasimov (Ed), Theoretical and experimental researches of high-speed body interactions (Tomsk State University, Tomsk, 2007) [in Russian]. 